\title{
Assessment of the Adherence of Primaquine for the Control and Elimination of Malaria in Senegal
}

\author{
Kiswendsida Thierry Guiguemde $e^{1,2, *}$, Yakou Dieye ${ }^{3}$, Babacar Faye ${ }^{1}$ \\ ${ }^{1}$ Department of Medical Parasitology, Medical Faculty, Cheikh Anta Diop University, Dakar, Senegal \\ ${ }^{2}$ Department of Parasitology and Mycology, Medical Faculty, Joseph Ki-Zerbo University, Ouagadougou, Burkina Faso \\ ${ }^{3}$ PATH, Malaria Control and Evaluation Partnership (MACEPA), Dakar, Senegal
}

Email address:

guiguemde@gmail.com (K. T. Guiguemde), ydieye@path.org (Y. Dieye), bfaye67@yahoo.fr (B. Faye)

${ }^{*}$ Corresponding author

\section{To cite this article:}

Kiswendsida Thierry Guiguemde, Yakou Dieye, Babacar Faye. Assessment of the Adherence of Primaquine for the Control and Elimination of Malaria in Senegal. American Journal of Biomedical and Life Sciences. Vol. 8, No. 5, 2020, pp. 185-188. doi: 10.11648/j.ajbls.20200805.17

Received: August 18, 2020; Accepted: August 27, 2020; Published: September 16, 2020

\begin{abstract}
Primaquine is a drug widely used for Plasmodium vivax malaria treatment. It is known to be active on Plasmodium falciparum mature gametocytes which are responsible for the transmission of the parasite from humans to mosquitoes. In 2018, Senegal initiated the large-scale use of a single low dose of primaquine associated with artemisinin-based combination therapy for malaria elimination. This study aimed to assess the therapeutic adherence of the implementation of primaquine in the Saint-Louis region. To measure therapeutic adherence, questionnaires were developed and made it possible to collect information from treated malaria patients, health workers, the investigating team and health authorities. Out of 1087 malaria patients, 585 received primaquine. Children under the age of 5 could not receive it due to the lack of the pediatric formulation. The involvement of private health facilities has shown its effectiveness in the implementation of the strategy. However, efforts must be made to improve the documentation of cases in the national surveillance system and to acquire logistical means facilitating the supply of the drug in remote locations. The authorities must become more involved in finding a solution for the availability and sufficient supply of primaquine to formulations adapted to the population.
\end{abstract}

Keywords: Malaria, Primaquine, Pediatric Formulation, Northern Senegal, Plasmodium falciparum

\section{Introduction}

The incidence of malaria has dropped significantly following the many advances made in the fight against this scourge in recent years [1]. Despite these efforts, much of the global malaria burden is attributed to the African continent. According to the World Health Organization (WHO), Africa alone recorded nearly $92 \%$ of malaria cases in 2016 and 2017 [1]. In Senegal, although malaria is still endemic in certain regions, the northern part of the country has recorded low incidences for a few years [2]. This new situation of very low transmission has led the National Malaria Control Program (NMCP) to stratify the districts based on the incidence, leading to adapt control actions according to local epidemiological characteristics [3]. Malaria is a real public health problem and its geographic spread is strongly caused by the transmission of the parasite to the vector. This transmission is due to the presence of the sexual forms of the parasite (gametocytes) in the blood which are easily transmitted to the vectors even at low densities $(<4$ gametocytes / $\mu \mathrm{l})$. Gametocytes are commonly found in persons treated and cured of malaria, which are reservoirs of the parasite and promote the transmission of malaria [4]. Primaquine is a molecule used for the treatment of Plasmodium vivax malaria which destroys mature gametocytes of Plasmodium falciparum $[4,5]$. Its use as a single low dose of $0.25 \mathrm{mg} / \mathrm{kg}$ in combination with Artemisinin-based Combination Therapy (ACTs) is recommended by WHO in areas of low transmission. This combination therapy offers possibilities to reduce the carriage of gametocytes and to interrupt the transmission of the parasite to vectors [6].

In Senegal the NMCP in partnership with the "Malaria Control and Elimination Partnership in Africa" (MACEPA) focused on the local elimination of $P$. falciparum malaria by 
adding the primaquine to standard treatment with ACTs. This new approach is centered on the northern part of the country and is the first step in the implementation of national malaria elimination. Since 2018, this therapeutic combination has been introduced in the Saint-Louis region with the involvement of all public and private health facilities. To appreciate the impact of this strategy on malaria elimination, it is important to evaluate the adhesion of the introduction of this molecule with the Standard treatment. Therapeutic adherence is a key element in the success of this therapeutic strategy. The objective of the present study was to assess patient compliance and healthcare staff adherence to the use of primaquine.

\section{Materials and Methods}

\subsection{Study Design and Area}

This was a descriptive cross-sectional study conducted in the Saint-Louis region in northern Senegal between January and December 2018. The northern part of Senegal is described as an area of low malaria transmission with lower incidence rates at $5 \%$. Malaria is transmitted mainly during the rainy season between August and October. It is a flood zone prone to flooding. The development of agriculture has led to an expansion of crops and increasing population pressure. The climate is Sahelian with a long dry season from November to June during which malaria transmission is maintained [7, 8]. The study was conducted in five health districts in the Saint-Louis region (Saint-Louis, Richard-Toll, Dagana, Pété and Podor).

\subsection{Study Population}

The patients who came for a consultation in one of the health facilities and in whom an RDT performed was positive, were recruited for the study and received a combination treatment of ACT and primaquine. Children under $30 \mathrm{~kg}$ did not receive primaquine.

\subsection{Study Settings}

Since 2018, Primaquine has been added in the treatment of uncomplicated malaria in five (5) health districts of the Saint-Louis region. All cases tested positive for malaria in public and private health facilities received one ACT (Arthemether + Lumefantrine, Artesunate + Amodiaquine or Dihydro-artemisinin + Piperaquine) and primaquine. Primaquine was administered at the low and single dose of $0.25 \mathrm{mg} / \mathrm{kg}$ with the first or second dose of ACT. Primaquine tablets were supplied in all health facilities in the region in formulations of $7.5 \mathrm{mg}$ and $15 \mathrm{mg}$ that cannot be broken.

After the diagnosis and treatment of the patient. The health worker communicates the patient's identification to the health district the same day by phone call or written message. Within three (3) days of notification, a team of investigators was deployed to the patient's home to collect the information about travel story, the perception of the taking of primaquine, the change in urine color and any side effects that occurred. The information was recorded in the District Health Information
Software2 (DHIS2) and Open Data Kit (ODK) system database. The surveys also concerned the health worker, the investigators as well as the staff of the NMCP.

\subsection{Analysis and Statistics}

The analysis concerned several factors which could influence the therapeutic adherence described by the WHO [9] as the factors related to the patient, the disease, the treatment and the care system. Data were entered by Excel software and analysed with the Epi-InfoTM 7 software (CDC, Atlanta, GA, USA) version 7.2.0.1. Descriptive statistics (central tendency, measures of spread, proportions, and percentages) were used to describe the data. Qualitative variables have been described in terms of size and percentage. Differences in proportions were analyzed with Chi square statistics and statistical significance was set at $\mathrm{p} \leq 0.05$.

\section{Results}

From January to December 2018, a total of 1087 malaria patients have been diagnosed and investigated in the region. the survey was carried out among 5 heads of investigative teams, 74 health personnel and a representative of the NMCP.

The mean age of the patients was $24.51 \pm 15.10$ years. Patients aged between 15 and 25 years were more represented $(30.73 \%)$ followed by those in the age group of 5 to 15 years $(25.39 \%)$. Men were more common $(72.31 \%)$ with a sex ratio of 2.61 .

Malaria cases were recorded in the 5 health districts of the region. The largest number of patients diagnosed with malaria came from the district of Saint-Louis $(36.52 \%)$, followed by the district of Pété (24.20\%) (Figure 1).

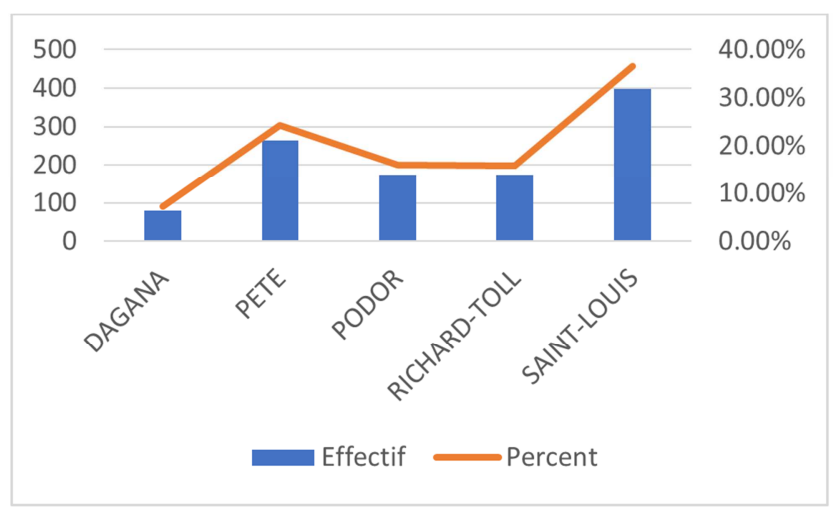

Figure 1. Distribution of malaria patients according to health districts in the Saint-Louis region in 2018.

Investigations showed that 697 patients $(64.12 \%)$ traveled outside their districts during the 2 weeks prior to diagnosis and 15 patients $(1.38 \%)$ traveled within the district where they slept at least one night.

Out of 1087 malaria cases recorded in the region, 585 patients received primaquine with an overall coverage rate of $54 \%$. No children under 5 years had received primaquine. Out of 276 children aged 5 to 15 years, primaquine was administered to only 82 children $(3.36 \%)$ with a minimum age 
of 7 years. Therapeutic coverage was unevenly distributed in the 5 health districts (Table 1) and was higher in the districts of
Saint-Louis (80.35\%) and Richard-Toll (66.47\%). Therapeutic coverage was lower in Dagana district (2.53).

Table 1. Distribution of therapeutic coverage and cases treated with ACT + primaquine by age and sex in the five districts of the Saint-Louis area in 2018.

\begin{tabular}{|c|c|c|c|c|c|c|}
\hline \multirow{2}{*}{ Ages } & \multicolumn{5}{|l|}{ Districts } & \multirow{2}{*}{ Total n (\%) } \\
\hline & Dagana n (\%) & Pete n (\%) & Podor n (\%) & Richard-Toll n (\%) & Saint-Louis n (\%) & \\
\hline$[0-05]$ & $0(0)$ & $0(0)$ & $0(0)$ & $0(0)$ & $0(0)$ & $0(0)$ \\
\hline [05-15] & $0(0)$ & $16(25.40)$ & $8(9.30)$ & $13(11.30)$ & $45(14.11)$ & $82(14.02)$ \\
\hline$[15-25]$ & $2(100)$ & $22(34.92)$ & $34(39.53)$ & $37(32.17)$ & $130(40.75)$ & $225(38.46)$ \\
\hline$[25-35]$ & $0(0)$ & $12(19.05)$ & $24(27.91)$ & $30(26.09)$ & $70(21.94)$ & $136(23.25)$ \\
\hline$[35-45]$ & $0(0)$ & $8(12.70)$ & $12(13.95)$ & $17(14.78)$ & $35(10.97)$ & $72(12.31)$ \\
\hline$[45-55]$ & $0(0)$ & $2(3.17)$ & $4(4.65)$ & $9(7.83)$ & $23(7.21)$ & $38(6.50)$ \\
\hline$[65-+]$ & $0(0)$ & $1(1.59)$ & $1(1.16)$ & $5(4.35)$ & $5(1.57)$ & $12(2.05)$ \\
\hline Total & $2(0.34)$ & $63(10.77)$ & $86(14.70)$ & $115(19.66)$ & $319(54.53)$ & $585(100)$ \\
\hline \multicolumn{7}{|l|}{ Sex } \\
\hline $\mathrm{F}$ & $1(50.00)$ & $23(36.51)$ & $21(24.42)$ & $20(17.39)$ & $58(18.18)$ & $123(21.03)$ \\
\hline M & $1(50.00)$ & $40(63.49)$ & $65(75.58)$ & $95(82.61)$ & $261(81.82)$ & $462(78.97)$ \\
\hline Total & $2(0.34)$ & $63(10.77)$ & $86(14.70)$ & $115(19.66)$ & $319(54.53)$ & $585(100)$ \\
\hline \multicolumn{7}{|c|}{ Primaquine therapeutic coverage } \\
\hline
\end{tabular}

The administration of primaquine was carried out by health personnel. Taking the treatment once allowed good compliance with the treatment. No patient reported a significant change in urine color. No case of acute hemolytic anemia has been reported. The urine color was dark yellow in $76.50 \%$ of the patients and light yellow in the others.

According to the clinical form, 967 cases $(88.96 \%)$ of uncomplicated malaria and 120 cases $(11.04 \%)$ of severe malaria were diagnosed in the region. Severe malaria was treated with injectable artesunate followed by oral treatment with ACT. Out of 120 cases of severe malaria treated parenterally, 46 patients $(7.86 \%)$ received primaquine with ACT relay therapy. Health workers reported that they did not know whether or not to give primaquine in these patients with severe malaria. The perception of the taking of primaquine is very good in the population. The absence of the pediatric formulation was deplored by health workers who had to exclude children under $30 \mathrm{~kg}$ from treatment. Health workers $(40.54 \%)$ reported shortages of primaquine supply. This break was also reported by 2 responsible investigators (Dagana and Pete teams). The involvement of the media made it possible to sensitize the population on the fight against malaria and to facilitate the acceptance of the introduction of primaquine in the treatment. The participation of customary chiefs and heads of Koranic schools facilitated popular support for the strategy. All the investigators reported logistical problems (motorbike, computer, telephones, etc.) for the investigation of patients who live more than $100 \mathrm{~km}$ from the health center and for the redeployment of primaquine tablets in places where there is a break. Lack of recording of information on taking primaquine was noted in Dagana district. Investigators report giving primaquine to patients without recording this information. Which has led to a very low rate of therapeutic coverage in this district.

According to the representative of NMCP, ordering primaquine for African countries presents many administrative difficulties. This order is being made in association with several countries expressing the need for primaquine. It is very difficult for the Senegal to order primaquine on its own and also to request a pediatric formulation of the molecule. All of the malaria patients received ACT. In the group of 585 patients who received primaquine, Arthemether-Lumefantrine was the most widely used ACT in combination with primaquine (99.25\%).

\section{Discussion}

The study revealed that primaquine can be used on a large scale and safely in the population of Senegal. The absence of the pediatric formulation has been an obstacle for the treatment of children under $30 \mathrm{~kg}$. This limit lowered the therapeutic coverage rate for primaquine. The supply disruptions in primaquine and inadequate logistics were observed in several points of benefit and have established limits for the effective implementation of the strategy. In the district of Dagana, treatment coverage was 2.53\%. Although investigators reported that they administered primaquine during treatment, these actions were not documented in the national surveillance system. This insufficient documentation of information on the use of primaquine has led to a significant drop in the rate of therapeutic coverage in this district. The concept of using primaquine in addition to ACTs on a large scale is new in Senegal and requires regular monitoring and supervision by health workers [10].

The involvement of customary and religious leaders and training schools is very important for sensitizing the population. The involvement of the various communication organs such as radio, television and concerts made it possible to sensitize the population on the fight against malaria and made it possible for the population to go easily to a health facility in case of fever. Arthemether-Lumefantrine is the most ACT used in Senegal. It has fewer side effects than Artesunate-Amodiaquine and exists in formulations of several strengths in tablet and can be adapted and administered to various age groups [11]. The involvement of private healthcare facilities was a determining factor in the identification and management of cases. Almost 50\% of diagnosed cases have been reported by this sector. The 
national malaria surveillance system has been applied to all levels of healthcare facilities (public and private) in order to manage and document all malaria cases in real time. This involvement made it possible to train all the actors involved in surveillance and to record a significant number of cases.

In the northern Senegal malaria is perceived as a disease imported by travelers (seasonal workers, drivers, travelers in transit, students returning from vacation). The movement of the population and asymptomatic carriers of the parasite make it difficult to control the transmission. The strategy of mass treatment with primaquine on a regional scale can be an effective weapon to considerably reduce gametocyte carriage [10]. This treatment is expensive and requires the support of partners and the country's authorities. Access to primaquine follows a group ordering process with other countries which does not allow the program to have a wide range on the quantity and the choice of the formulation of the molecule. Advocacy should be made to facilitate the ordering of primaquine in sufficient quantity and in a formulation adapted to the needs of the population.

As countries move towards the elimination of malaria, the residual foci of transmission are defined with precise geographical limits revealing the problem of the importation of malaria which is gaining importance [11]. The use of primaquine in cross-border and interdistrict case management would control the importation of malaria and block the transmission of the disease [12].

\section{Ethical Approval}

The study protocol was submitted for approval to Senegal's National Committee for Ethics for Health Research (CNERS) (Reference: 046/2015/CER/UCAD). All participants were informed of the study and their consent was obtained.

\section{Funding}

This work was supported by MACEPA and the NMCP of Senegal.

\section{Conflict of Interest}

The authors declare that they have no conflict of interest.

\section{Acknowledgements}

The authors thank the entire MACEPA and NMCP team, the administrative and community leaders of the Saint-Louis region for their involvement in the smooth implementation.

\section{References}

[1] WHO. World Malaria Report 2017. World Health Organization. Geneva: WHO, 2017.

[2] R. C. Tine, K. Sylla, B. Faye B, E. Poirot, F. B. Fall, D. Sow, et al. Safety and efficacy of adding a single low dose of primaquine to the treatment of adult patients with Plasmodium falciparum malaria in Senegal, to reduce gametocyte carriage: a randomized controlled trial. Clinical Infectious Diseases, 2017; 65 (4): 535-43.

[3] Ministry of Health, Prevention and Social Action. National Strategic Plan to Fight Malaria in Senegal 2016 - 2020. National Program to Fight Malaria. 2015. K. Elissa, "Title of paper if known," unpublished.

[4] F. Santolamazz, P. Avellino, G. Siciliano, F. A. Yao, F. Lombardo, J. B. Ouédraogo, V. D. Mangano. Detection of Plasmodium falciparum male and female gametocytes and determination of parasite sex ratio in human endemic populations by novel, cheap and robust RTqPCR assays. Malar J. 2017; 16 (1), 468. doi: 10.1186/s12936-017-2118-z.

[5] B. Greenwood and R. Tine. Primaquine to stop transmission of falciparum malaria. Lancet Infectious Diseases, 2016; 16 (6), 623-624. doi: 10.1016/s1473-3099(15)00550-2.

[6] WHO. Evidence Review Group: The Safety and Effectiveness of Single Dose Primaquine as a P. falciparum gametocytocide. WHO, Geneva: 2013.

[7] A. B. Sagna, L. Gaayeb, J. B. Sarr, S. Senghor, A. Poinsignon, S. Boutouaba-Combe, et al. Plasmodium falciparum infection during dry season: IgG responses to Anopheles gambiae salivary gSG6-P1 peptide as sensitive biomarker for malaria risk in Northern Senegal, Malar. J., 12 (1) (2013) 301. doi https://doi.org/10.1186/1475-2875-12-301.

[8] O. Faye, O. Gaye, J. F. Molez, D. Fontenille, L. Konate, J. P. Hervé, et al. Aménagements hydro-agricoles et paludisme: Étude comparative d'une zone rizicole et d'une zone de culture, in: J. P. Hervé, J. Brengues (Eds.), Aménagements hydro-agricoles et santé (vallée du fleuve Sénégal), Paris, ORSTOM, 1998, pp. 283-291. http://www.documentation.ird.fr/hor/fdi:010014352 accessed 9/4/2018.

[9] WHO. Adherence to Long-Term Therapies: Evidence for Action. Switzerland, World Health Organization, 2003, 211 p. https://apps.who.int/iris/bitstream/handle/10665/42682/92415 45992.pdf?sequence $=1$ accessed 9/1/2020.

[10] S. V. Dlamini, R. J. Kosgei, N. Mkhonta, Z. Zulu, K. Makadzange, S. Zhou, P. Owiti, et al. Case management of malaria in Swaziland, 2011-2015: on track for elimination?. $\begin{array}{lllll}\text { PHA } & 2018 ; & 8 & \text { (S1): } & \text { S3-S7. }\end{array}$ http://dx.doi.org/10.5588/pha.17.0047.

[11] W. R. Taylor, H. K. Naw, K. Maitland, T. N. Williams, M. Kapulu, U. D'Alessandro, et al. Single low-dose primaquine for blocking transmission of Plasmodium falciparum malaria a proposed model-derived age-based regimen for sub-Saharan Africa. BMC Medicine. 2018; 16 (1): 11.

[12] J. M. Cohen, S. Dlamini, J. M. Novotny, D. Kandula, S. Kunene, A. J. Tatem. Rapid case-based mapping of seasonal malaria transmission risk for strategic elimination planning in Swaziland. Malar J. 2013; $12 \quad$ (1): 61. https://doi.org/10.1186/1475-2875-12-61.

[13] T. Yangzom, C. S. Gueye, R. Namgay, G. N. Galappaththy, K. Thimasarn, R. Gosling, et al. Malaria control in Bhutan: case study of a country embarking on elimination. Malar J. 2012; 11 (1): 9 . 